\title{
A Superconducting Cold-Electron Bolometer with SIS' and Josephson Tunnel Junctions
}

\author{
Leonid Kuzmin
}

Received: 22 July 2007 / Accepted: 15 September 2007 / Published online: 19 January 2008

(C) Springer Science+Business Media, LLC 2008

\begin{abstract}
A novel concept of a Superconducting Cold-Electron Bolometer (SCEB) with Superconductor-Insulator-Weak Superconductor (SIS') Tunnel Junction and Josephson Junction has been proposed. The main innovation of this concept is utilizing the Josephson Junction for DC and HF contacts, and for thermal isolation. The SIS' junction is used also for electron cooling and dc readout of the signal. The SIS' junction is designed in loop geometry for suppression of the critical current by a weak magnetic field. The key moment of this concept is that the critical current of the Josephson junction is not suppressed by this weak magnetic field and can be used for de contact. Due to this innovation, a robust two layer technology can be used for fabrication of reliable structures. A direct connection of SCEBs to a 4-probe antenna has been proposed for effective RF coupling.
\end{abstract}

Keywords Cold-electron bolometer · SIS' tunnel junction · Josephson junction • JFET readout

PACS $85.25 . \mathrm{Pb} \cdot 74.50 .+\mathrm{r} \cdot 74.25 . \mathrm{Bt}$

\section{Introduction}

Recent Cosmology experiments have discovered that the Universe consists mainly of mysterious Dark Energy and Dark Matter [1]. Indeed, in 2006, a Nobel Prize was awarded for the experimental observation of anisotropies in the Cosmic Microwave Background $(\mathrm{CMB})$ radiation, and the subsequent realization that the expansion of the Universe is controlled by unknown forces [2]. There are several cosmology instruments (BOOMERanG, OLIMPO, B-POL, CLOVER, ...) that are being designed

\footnotetext{
L. Kuzmin (ه)

Chalmers University of Technology, 41296 Gothenburg, Sweden

e-mail: leonid.kuzmin@mc2.chalmers.se
} 


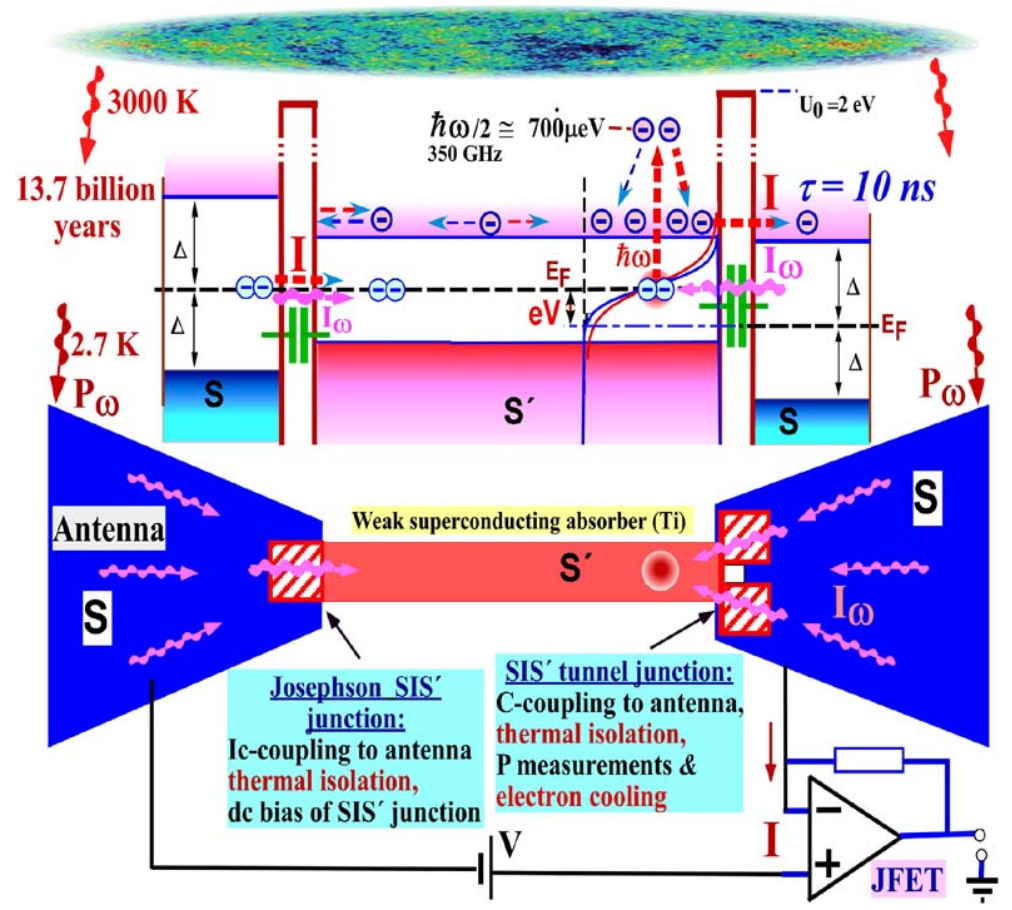

Fig. 1 (Color online.) Schematic of a Superconducting Cold-Electron Bolometer (SCEB) with SIS' and Josephson Tunnel Junctions and a JFET readout. The SIS' junction is used for capacitive coupling to the antenna, thermal isolation, electron cooling and dc readout by a JFET. The Josephson junction is used for $\mathrm{dc}$ and hf contacts and for thermal isolation

to measure anisotropies and the $B$-polarization in the $\mathrm{CMB}$ in order to detect gravity waves in the early moments of the Big Bang. Accurate measurement of the CMB should be done using a new generation of sensitive detectors.

An ultra-sensitive Cold-Electron Bolometer (CEB) [3] is one of the promising candidates for these experiments. The CEB concept is based on direct electron cooling of the absorber and provides high sensitivity and high saturation power. The CEB concept has been accepted as the main detector for $350 \mathrm{GHz}$ channel of BOOMERanG [4]. The main requirement is to develop a CEB array for 92 channels with high polarization resolution. The NEP with JFET readout should be less than photon noise for optical power load of $10 \mathrm{pW}$.

A novel concept of a Superconducting Cold-Electron Bolometer (SCEB) with SIS' and Josephson tunnel junctions has been proposed. The main innovation in comparison with the CEB with double SIN junction [3], CEB with SIN and Andreev contact [5] and SCEB with double SIS' junction [6, 7] is effective utilizing the Josephson Junction for dc and HF contacts and for thermal isolation. The SIS' junction (for HF coupling, thermal isolation, electron cooling and dc readout) is proposed in loop geometry for suppression of the critical current by a weak magnetic field. Striking moment of the concept is that the critical current of Josephson junction is not suppressed by this weak magnetic field. As a result of this innovation, a robust two 

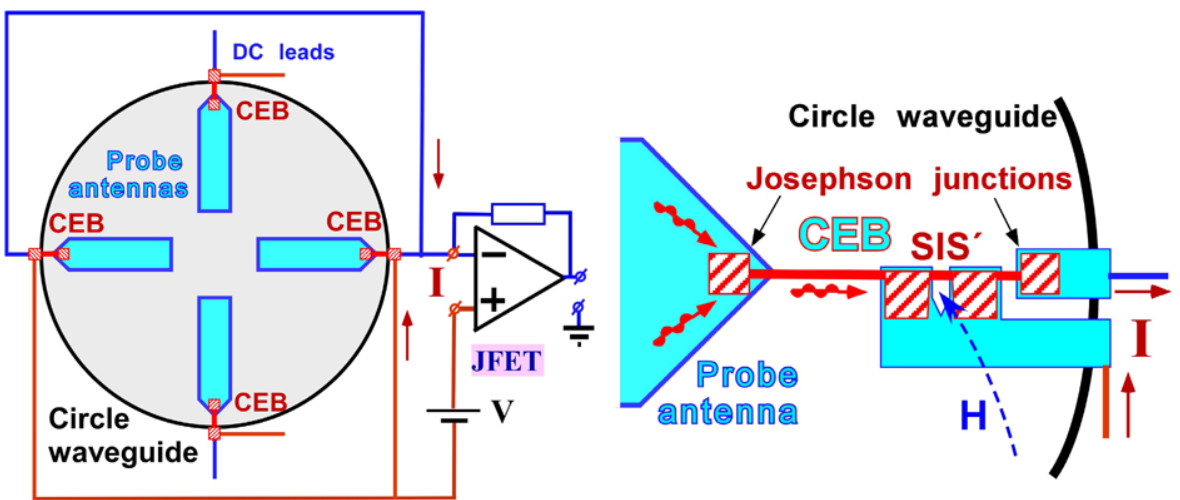

Fig. 2 (Color online.) (Left) Schematic of CEBs connected directly to a 4-probe antenna in a circle waveguide. (Right) Fragment of the CEB connection to a probe antenna with an additional Josephson junction for dc bias supply

layer technology can be used for fabrication of both SIS' and Josephson tunnel junctions. In this paper we analyze a realization of the SCEB for the $350 \mathrm{GHz}$ channel of BOOMERanG and other Cosmology instruments.

\section{Model}

Here we analyze a system with the most effective direct connection of SCEBs to 4-probe antenna in circle waveguide (Fig. 2a). In contrast to a previous concept of SCEBs with coplanar lines [7], the HF region is limited by circle waveguide area. The optimal point for insertion is connection of the probe to the waveguide (maximum HF current). The problem of DC bias of an SIS' junction could be decided by introducing one more Josephson junction at the right end of the absorber (Fig. 2b). Two opposite SCEBs are connected in parallel for each polarization by dc leads and measured by a JFET readout in voltage-biased mode. The optimal bias point of SIS' junction is between difference and sum gaps where the I-V curve has increased dynamic resistance. For the JFET noise, $3 \mathrm{nV} / \mathrm{Hz}^{1 / 2} \& 5 \mathrm{fA} / \mathrm{Hz}^{1 / 2}$, the effective noise impedance is around $600 \mathrm{KOhm}$. The suppression of the JFET voltage noise is important for this realization.

Current fluctuations of JFET are rather low at the level of $5 \mathrm{fA} / \mathrm{Hz}^{1 / 2}$.

For analysis we use a previous concept of the CEB with strong electrothermal feedback due to electron cooling [3, 7-9]. The operation of CEB can be analyzed using heat balance equation [9]:

$$
P_{\text {cool }}\left(V, T_{e}, T_{p h}\right)+P_{e-p h}\left(T_{e}, T_{p h}\right)+C_{\Lambda} \frac{d T}{d t}=P_{0}+\delta P(t) .
$$

Here, $P_{e-p h}\left(T_{e}, T_{p h}\right)$ is the heat flow from electron to phonon subsystems in the absorber, $T_{e}$ and $T_{p h}$ are the electron and phonon temperatures of the absorber; $P_{\text {cool }}\left(V, T_{e}, T_{p h}\right)$ - cooling power of the SIS' tunnel junction; $C_{\Lambda}=\Lambda \gamma T_{e}$ is the specific heat capacity of the absorber, $\Lambda$-a volume of the absorber; $P(t)$ - the incoming 
RF power. The sensitivity of the device is characterized by the current responsivity $S_{I}$ on the incoming power,

$$
S_{I}=\frac{\partial I_{\omega}}{\partial P_{\omega}}=\frac{\partial I / \partial T}{G_{c o o l}+G_{e-p h}+i \omega C_{\Lambda}},
$$

where $G_{\text {cool }}=\partial P_{\text {cool }} / \partial T$, is the cooling thermal conductance of the SIS' junction and $G_{e-p h}=\partial P_{e-p h} / \partial T$ is electron-phonon thermal conductance of the absorber. Noise properties are characterized by the NEP

$$
N E P_{\text {total }}^{2}=N E P_{e-p h}^{2}+N E P_{S I S}^{2}+\frac{\delta I^{2}}{S_{I}^{2}} .
$$

Here $N E P_{e-p h}^{2}$ is the noise due to electron-phonon interaction; $N E P_{S I S}^{2}$, is the noise of an SIS' junction and $\delta I 2 / S 2_{I}$ is the noise of an JFET amplifier.

The noise of the SIS' junction has three components: shot noise $2 e I / S_{I}^{2}$, the heat flow noise and the anticorrelation term between these two processes $[9,10]$

$$
N E P_{S I S}^{2}=\delta P_{\omega}^{2}-2 \frac{\delta P_{\omega} \delta I_{\omega}}{S_{I}}+\frac{\delta I_{\omega}^{2}}{S_{I}^{2}} .
$$

This anticorrelation is a form of the electrothermal feedback discussed earlier by Mather [11]. For superconductor absorber with concentration of electrons just near the gap this anticorrelation is very strong and could lead, in first approximation, to almost $100 \%$ compensation of the shot noise:

$$
\delta P_{\omega}^{2}=2 \Delta P_{0}, \quad \delta I_{\omega}^{2}=2 e^{2} P_{0} / \Delta, \quad S_{I}=e / \Delta, \quad N E P_{S I S}^{2} \cong 0 .
$$

For every chosen voltage we first solve the heat balance equation, find the electron temperature in the absorber taking into account the effect of the electron cooling, and then determine current responsivity and NEP.

\section{Results and Discussion}

For an optical power load of $P_{0}=5 \mathrm{pW}$ per polarization for the $350 \mathrm{GHz}$ channel, the photon noise is $N E P_{\text {phot }}=\sqrt{2 P_{0} \times h f}=4.3 \times 10^{-17} \mathrm{~W} / \mathrm{Hz}^{1 / 2}$.

Figure 3 shows simulation of the different contributions to the total NEP of the detector for an optimized geometry of the bolometer. We see that for a range of bias voltage from $170 \mu \mathrm{V}$ to $210 \mu \mathrm{V}$, the total NEP of the SCEB is well below the photon noise: $N E P_{\text {tot }}<N E P_{\text {phot }}$. The range of voltages less than $170 \mathrm{GHz}$ is not recommended for use because, due to negative slope the IV-curve, the operation point would be unstable. In addition, the $N E P_{\text {tot }}$ of the SCEB is dominated by the shot/heat noise of the detector $\left(N E P_{S I S}\right)$ corresponding to the background limited mode of operation.

Figure $3 b$ illustrates the effect of the noise reduction of SIS' tunnel junction. The figure shows all components of SIS' noise: $N E P_{\text {shot }}, N E P_{\text {heat }}$ and correlation term 

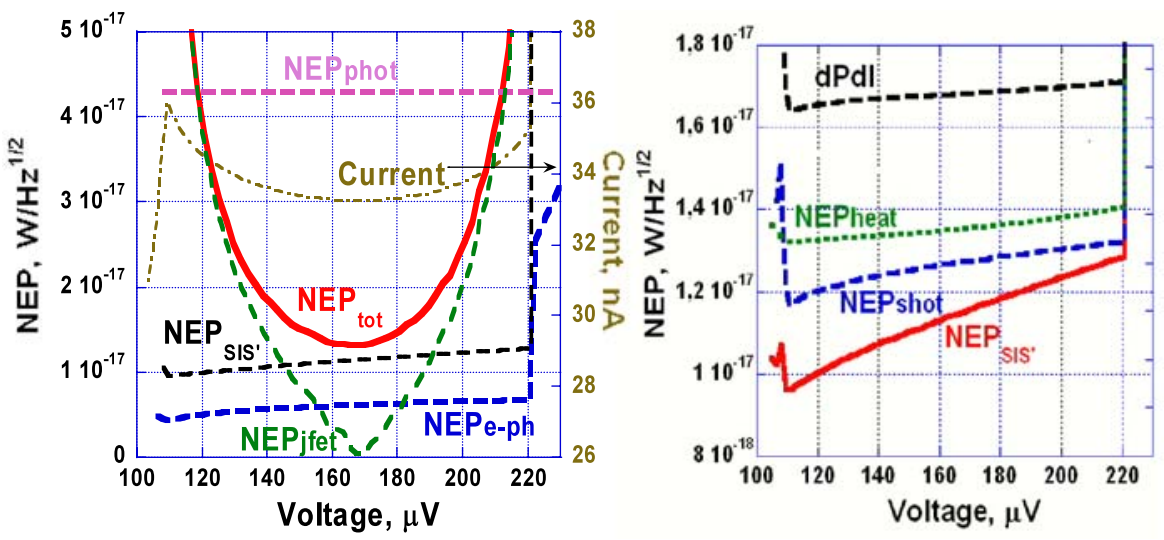

Fig. 3 (Color online.) (Left) NEP components of the SCEB for $I_{J F E T}=5 \mathrm{fA} / \mathrm{Hz}^{1 / 2}, V_{J F E T}=3$ $\mathrm{nV} / \mathrm{Hz}^{1 / 2}, R=0.2 \mathrm{kOhm}, \Lambda=0.04 \mu \mathrm{m}^{3}$. The $N E P_{\text {tot }}$ is less than $N E P_{\text {phot }}$; (Right) Resulting SIS' junction noise with strong cancellation of the $N E P_{\text {shot }}$ and $N E P_{\text {heat }}$ due to anticorrelation term dPdI between them
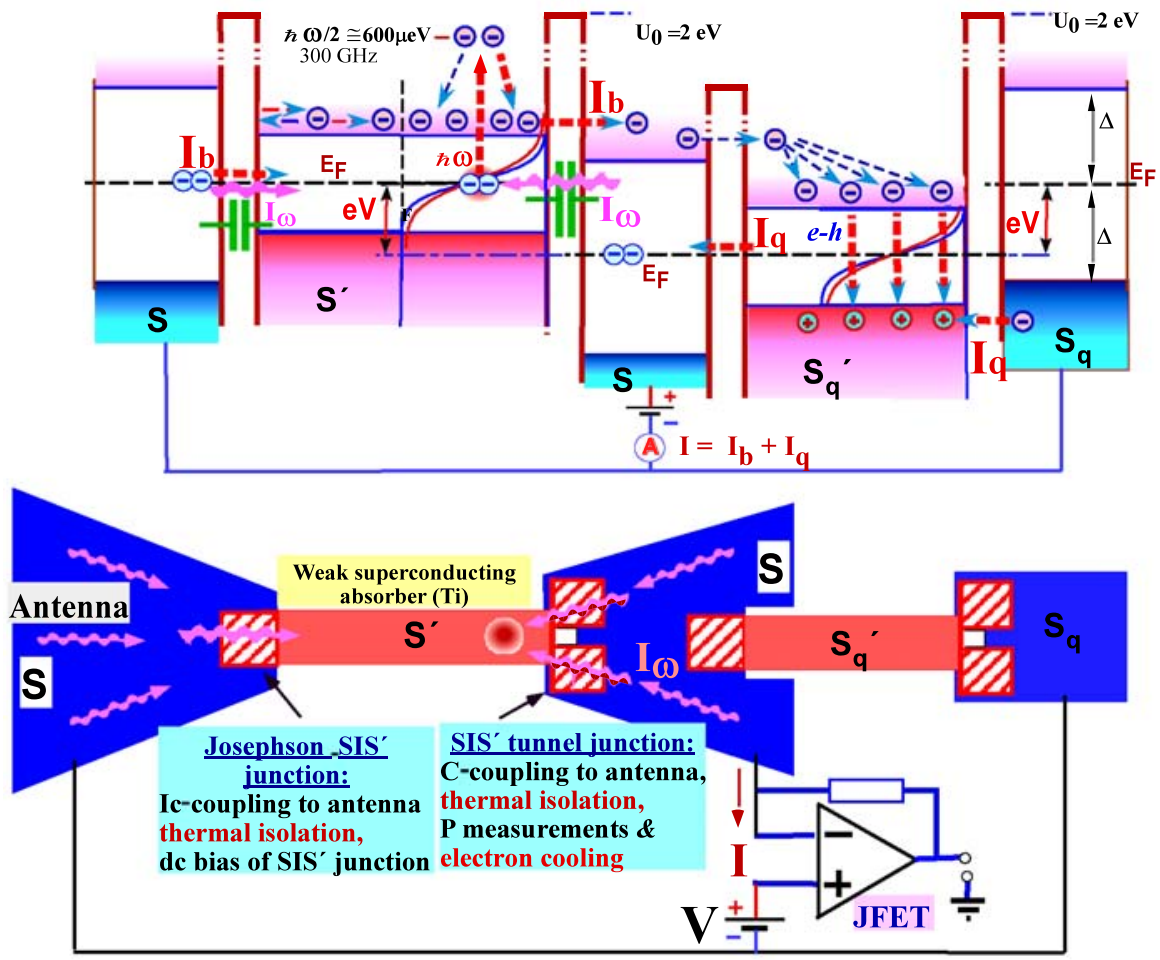

Fig. 4 (Color online.) Cascade Quasiparticle Amplifier (QPA) based on SIS' and Josephson junctions. The top diagram shows principle of operation of the QPA 
$(\mathrm{dPdI})^{1 / 2}$. The final noise, $N E P_{S I S}$, is clear less than original noise components. The effect is stronger than for SIN junction noise [9] due to well-defined level of quasiparticle energy just near the gap.

If we have still domination of the noise of JFET amplifier, a cascade quasiparticle amplifier (QPA) ${ }^{12}$ can be used to increase further the output current (responsivity) and decrease contribution of the amplifier. Typical schematic of the QPA is shown in Fig. 4. The basic principle of the QPA is based on catching the hot quasiparticles in strong superconductor $\mathrm{S}$ by a weak superconductor Sq' through the Josephson tunnel junction. The energy of quasiparticles in $\mathrm{S}$ will be released in Sq' creating proportional number of quasiparticles. After transformation of quasiparticles to holes in S' due to inelastic e-e interaction, the quasiholes will be readout by right tunnel junction and current Iq will be added to the main current I.

The coefficient of amplification can be estimated from the diagram in Fig. 4 as

$$
K=\frac{I-I q}{I}=1+\frac{\Delta+\Delta^{\prime} * 1.5}{\Delta^{\prime}} \cong 5 \text { for } \mathrm{Al}(1.2 \mathrm{~K}) \text { and } \mathrm{Ti}(0.5 \mathrm{~K}) .
$$

For larger K we should select strong superconductor with larger gap or weaker superconductor for quasiparticle trap. In contrast to realization of QPA with SIN tunnel junction and Andreev contact [12] the S' and Sq' are made in the same layer that would considerably improve technology.

Acknowledgements The author would like to thank Paolo de Bernardis for request on CEBs for BOOMERanG project stimulated this work and Dmitri Golubev, Philip Mauskopf and Michael Tarasov for interesting discussions. The work was supported by VR and STINT Swedish agencies.

\section{References}

1. C. Seife, Science 302, 2038 (2003)

2. 2006 Nobel Prize in Physics for discovery of the blackbody form and anisotropy of the cosmic microwave background radiation

3. L. Kuzmin, in Proc. of SPIE Conference "Millimeters and Submillimeter Detectors", vol. 5498, p. 349, Glasgow, June 2004

4. BOOMERANG_-balloon telescope: Measurements of CMB Polarization, http://oberon.roma1.infn. it/boomerang/b2k/

5. L. Kuzmin, in Proc. of the 17th Int. Symp. on Space Terahertz Technol., ISSTT-2006, Paris, May 2006

6. L. Kuzmin, Microelectron. Eng. 69, 309 (2003)

7. L. Kuzmin, P. Mauskopf, D. Golubev, J. Phys. Conf. Ser. (JPCS) 43, 1298-1302 (2006)

8. L. Kuzmin, D. Golubev, Physica C 372-376, 378 (2002)

9. D. Golubev, L. Kuzmin, J. Appl. Phys. 89, 6464 (2001)

10. S. Golwala, J. Johum, B. Sadoulet, in Proc. of the 7 International Workshop on Low Temperature Detectors, pp. 64-65. Munich, July 1997

11. J.C. Mather, Appl. Opt. 21, 1125 (1982)

12. L. Kuzmin, Physica C 372-376, 457 (2002) 